Document downloaded from:

http://hdl.handle.net/10251/53510

This paper must be cited as:

Oscar Carranza Castillo; Figueres Amorós, E.; Gabriel Garcerá; Luis Gerardo González Morales (2011). Comparative study of speed estimators with highly noisy

measurement signals for Wind Energy Generation Systems. Applied Energy. 88:805-813. doi:10.1016/j.apenergy.2010.07.039.

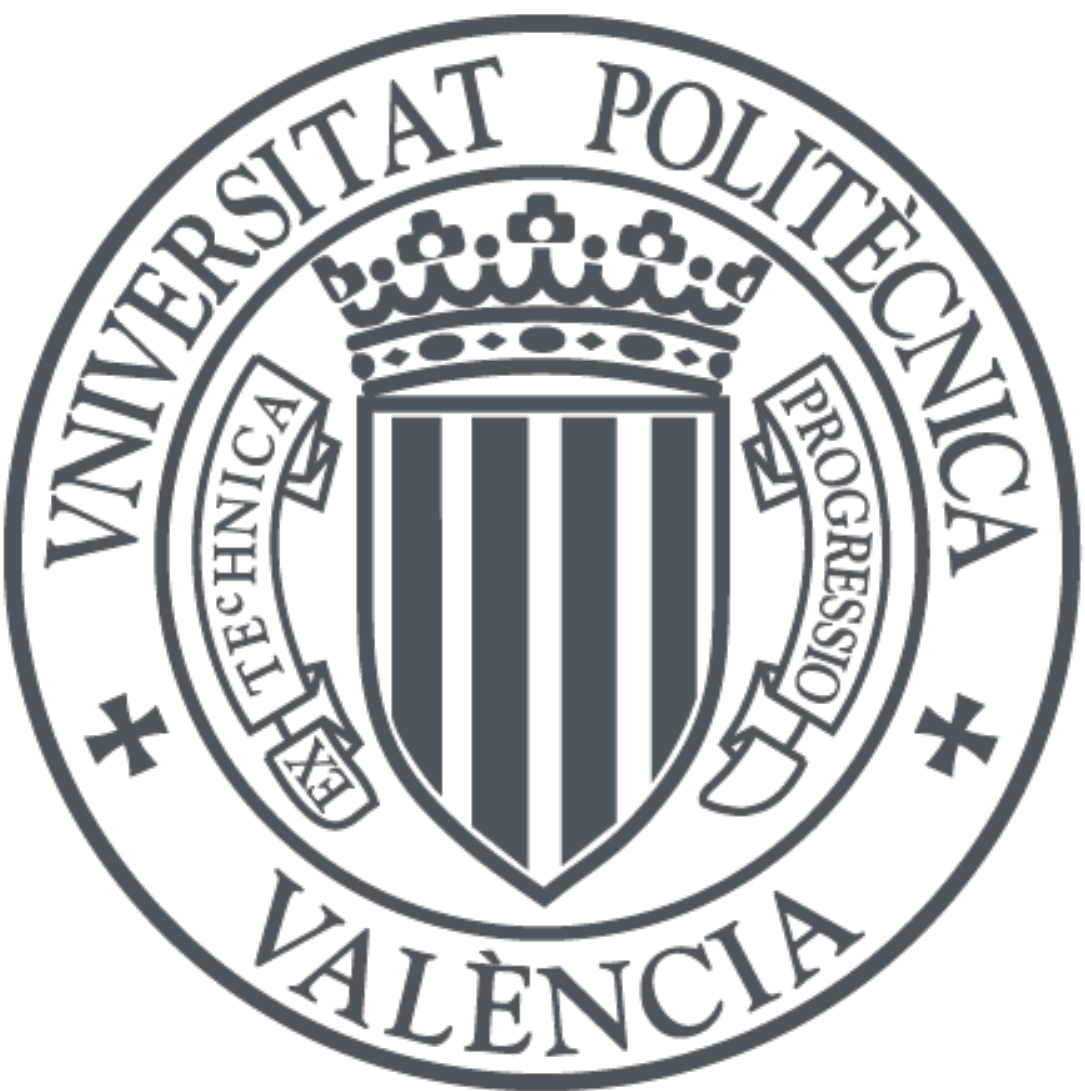

The final publication is available at

http://dx.doi.org/10.1016/j.apenergy.2010.07.039

Copyright Elsevier

Additional Information 


\title{
Comparative study of Speed Estimators with highly Noisy Measurement Signals for Wind Energy Generation Systems
}

\author{
O. Carranza ${ }^{1}$, E. Figueres ${ }^{2}$, G. Garcerá ${ }^{2}$, L. G. Gonzalez ${ }^{3}$ \\ ${ }^{1}$ Escuela Superior de Cómputo, Instituto Politécnico Nacional, \\ Av. Juan de Dios Batiz S/N, Col. Lindavista, Del. Gustavo A. Madero, \\ 07738, D.F., México, email ocarranzac@ipn.mx, \\ ${ }^{2}$ Grupo de Sistemas Electrónicos Industriales, Departamento de Ingeniería Electrónica, \\ Universidad Politécnica de Valencia, Camino de Vera S/N, 7F, 46020, \\ Valencia, Spain, http://www.gsei.upv.es, email: efiguere@eln.upv.es, ggarcera@eln.upv.es, \\ ${ }^{3}$ Departamento de Ingeniería Electrónica, Universidad de los Andes, \\ Merida, Venezuela, email: Igerardo@ula.ve
}

\begin{abstract}
This paper presents a comparative study of several speed estimators to implement a sensorless speed control loop in Wind Energy Generation Systems driven by power factor correction threephase boost rectifiers. This rectifier topology reduces the low frequency harmonics contents of the generator currents and, consequently, the generator power factor approaches unity whereas undesired vibrations of the mechanical system decrease. For implementation of the speed estimators, the compared techniques start from the measurement of electrical variables like currents and voltages, which contain low frequency harmonics of the fundamental frequency of the wind generator, as well as switching frequency components due to the boost rectifier. In this noisy environment it has been analyzed the performance of the following estimation techniques: Synchronous Reference Frame Phase Locked Loop, speed reconstruction by measuring the dc current and voltage of the rectifier and speed estimation by means of both an Extended Kalman Filter and a Linear Kalman Filter.
\end{abstract}

Keywords: Distributed Generation, Sensorless speed control, Wind Energy Generation Systems.

\section{Introduction}

Wind Energy is one of the most promising renewable sources, not only in the Megawatt power range, but also in the case of small generators up to several kW. Small wind generators may be individually used, or in combination with other sources like photovoltaic panels, to take profit of the distributed generation concept. A Wind Energy Generation System (WEGS) is composed by an electrical generator which is driven by a power converter. In the low power range, Permanent Magnet Synchronous Generators (PMSG) [1] are usually preferred. A WEGS based on PMSG operates at variable speed, so that both amplitude and frequency of the generator output voltage vary in a certain range, following the wind speed [2]. Therefore, the power converter plays a double role. On one hand, it processes the energy that the generator produces, by injecting it into an electrical grid or by charging the batteries in stand-alone systems. On the other hand, the power converter is controlled by means of a Maximum Power Point Algorithm (MPPT) [3], so that the wind turbine can extract as maximum energy as possible from the wind.

Several topologies of power converters to drive PMSG have been proposed [1]. In [4] a ThreePhase Boost Rectifier operating in Discontinuous Conduction Mode (DCM) with Peak Current Mode Control (PCC) was presented. With such a topology, the PMGS may be operated at power factors approaching unity, whereas the low frequency harmonics of the generator currents significantly decrease when compared to the Continuous Conduction Mode of operation.

Fig. 1 shows the scheme of a low power WEGS based on a PMSG and a Three-Phase Boost Rectifier operating in discontinuous conduction mode. Grid connection of the WEGS is considered in this paper, so that the rectifier feeds the DC-link of a grid-connected power inverter. The control 
of the inverter stage is out of the scope of this paper, but details about this issue may be found in [5]-[6]. A current control loop regulates the PMSG torque, which directly depends on the generator currents and therefore on the dc current of the rectifier. After closing the current loop, a speed control loop regulates the PMSG following a reference signal that is calculated by the MPPT algorithm. To obtain the feedback signal for the speed control loop, it is possible to mount speed sensors on the shafts of the PMSG (resolvers, encoders or Hall-effect sensors). However, the use of these sensors increases the complexity, weight and cost of the system. Due to the fact that both the voltages and currents of the PMSG may be expressed as a function of the mechanical speed, the latter can be estimated from measurement of the electrical quantities, eliminating the need for mechanical sensors. This technique is known as sensorless speed control of the PMSG and was applied to WEGS driven by a back-to-back converter in [7]. The problem in developing speed estimators for the WEGS studied in this paper is that the measured signals contain low frequency harmonics of the fundamental frequency of the wind generator, as well as switching frequency components due to the boost rectifier. Furthermore, the fundamental and its low frequency harmonics have different values depending on the generator speed. This situation is depicted in Fig. 2, where it may be seen the aspect of both the current and the voltage in one phase of the PMSG for several speed operation points.

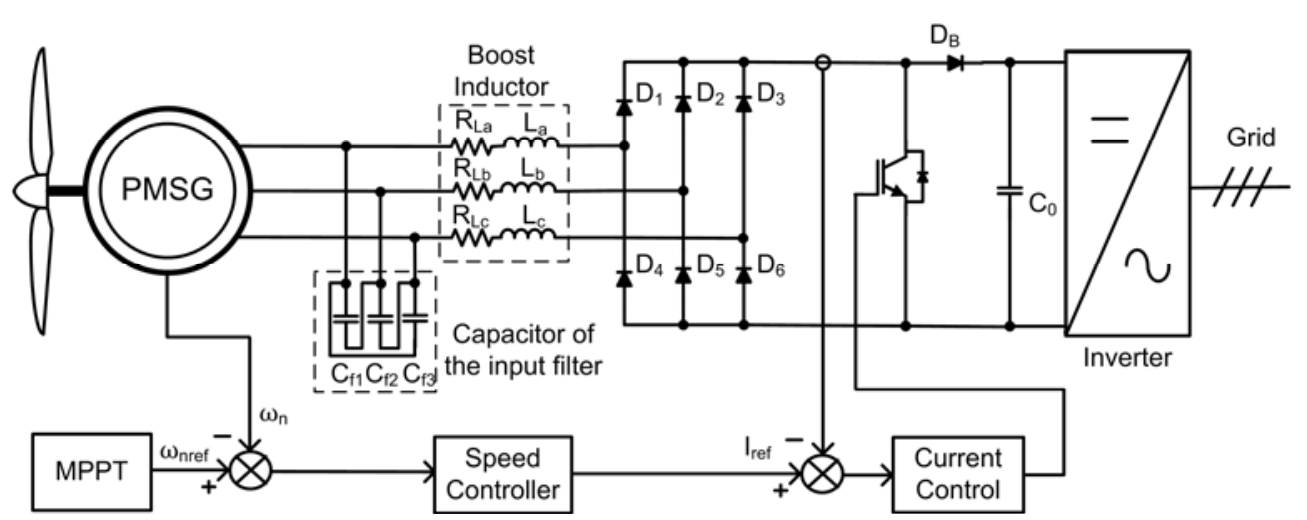

Fig. 1. Scheme of a Boost Rectifier with Speed control
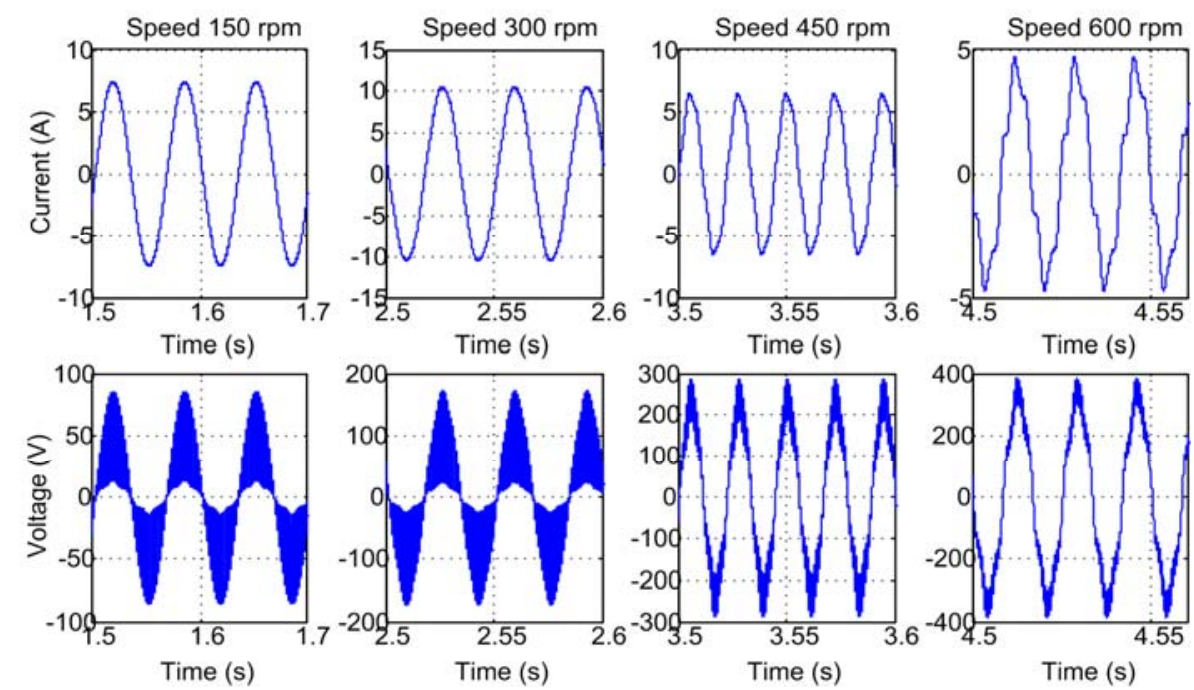

Fig. 2. Current and Voltage in one phase of the PMSG controlled by a boost rectifier working in discontinuous conduction mode

The performance of the following speed estimation methods is studied in this work: 1) synchronous reference frame Phase Locked Loop, 2) speed estimation by measuring dc current and voltage of the rectifier, 3) speed estimation using an Extended Kalman Filter, and 4) speed estimation using a Linear Kalman Filter.

TABLE I shows the parameters of the system under study. 
TABLE I. The values of the simulated system.

\begin{tabular}{lc}
\hline \multicolumn{1}{c}{ Characteristics } & Values \\
\hline Output voltage range of the generator $\left.\left(V_{a b}=V_{b c}=V_{c a}\right)\right)$ & $104-416 V_{r m s}$ \\
Constant of the electromotive force $\left(K_{f e m}\right)$ & $6.63 V_{r m s} / \mathrm{rad} / \mathrm{s}$ \\
Number of poles $\left(n_{p}\right)$ & 12 \\
Speed range of the generator $\left(n_{m}\right)$ & $150-600 \mathrm{rpm}$ \\
Angular Speed range of the generator $\left(\omega_{m}=\pi \cdot n_{m} / 30\right)$ & $15.7-62.83 \mathrm{rad} / \mathrm{s}$ \\
Angular frequency range of the generator $\left(\omega_{e}=\omega_{m} \cdot n_{p} / 2\right)$ & $94.2-376.98 \mathrm{rad} / \mathrm{s}$ \\
Inductance of one phase of the generator $\left(L_{g a}=L_{g b}=L_{g c}=L_{g}\right)$ & $25 \mathrm{mH}$ \\
Resistance of one phase of the generator $\left(R_{L g a}=R_{L g b}=R_{L g c} \equiv R_{g}\right)$ & $5 \Omega$ \\
Boost Inductance associated to each phase $\left(L_{a}=L_{b}=L_{c} \equiv L\right)$ & $375 \mu \mathrm{H}$ \\
Resistance in series associated to the Boost inductor in each & $37.5 \mathrm{~m} \Omega$. \\
phase $\left(R_{L a}=R_{L b}=R_{L c} \equiv R_{L}\right)$ & $2.2 \mu \mathrm{F}$ \\
Filter Capacitors $\left(C_{f 1}=C_{f 2}=C_{f 3}\right)$ & $5 \mathrm{kHz}$ \\
Switching frequency $\left(f_{s w}\right)$ & $10 \mu \mathrm{s}$ \\
Sampling time $\left(T_{S}\right)$ & \\
\hline
\end{tabular}

\section{Speed estimation using a Phase Locked Loop}

The first speed estimation method under study uses a Phase Locked Loop (PLL) based on the synchronous reference frame (SRF) [8]-[9], following the scheme shown by Fig. 3. The threephase system produced by the PMSG is transformed into the $d q$ synchronous reference frame by using the Park transformation. The angular position of this $d q$ reference frame is controlled by a feedback loop which controls the q component of the PMSG voltage to be zero $\left(V_{q}=0\right)$.

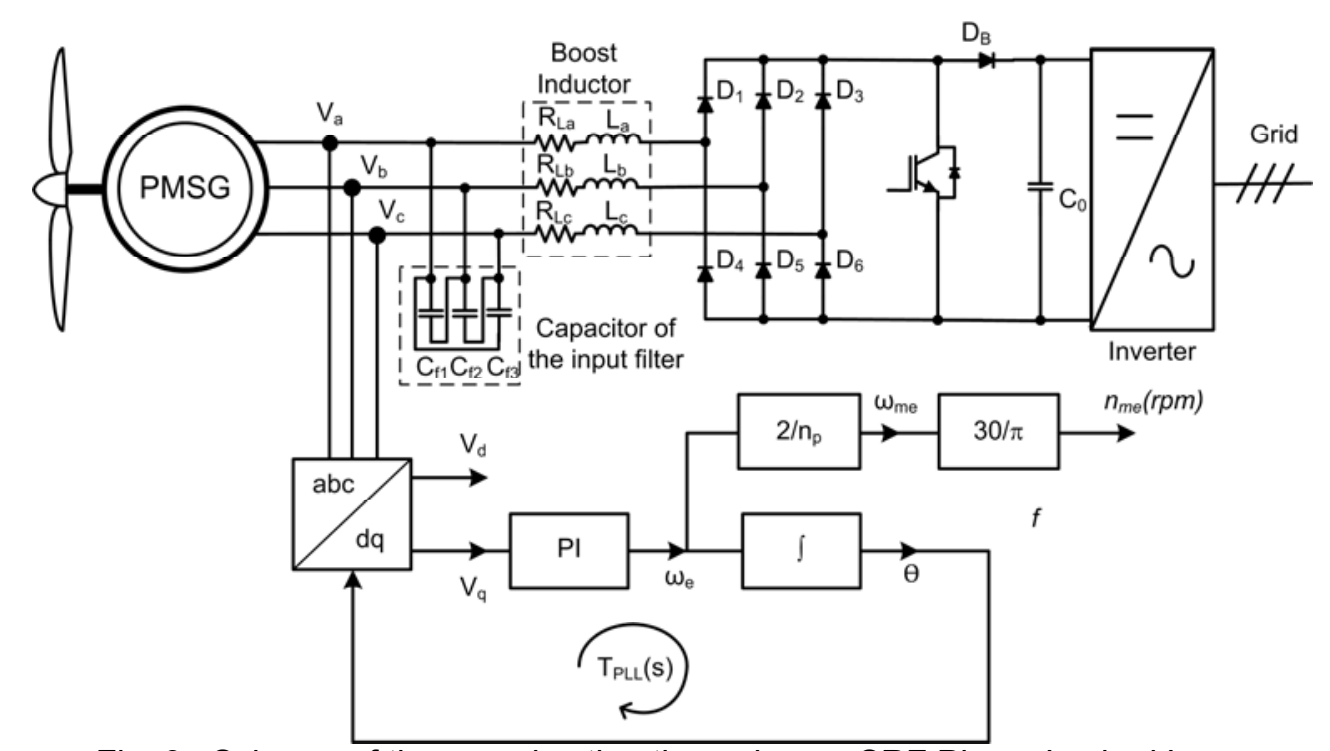

Fig. 3. Scheme of the speed estimation using an SRF Phase Locked Loop

The loop gain transfer function to analyze the PLL stability, $T_{P L L}$, is determined by (1):

$$
T_{P L L}(s)=V_{d} \cdot P I \cdot \frac{1}{s}
$$

$V_{d}$ is the magnitude of the d-component of the PMSG voltage in the synchronous reference frame, and $P I$ is the transfer function of a simple proportional-integral controller. Given the generator speed range $n_{m}=150$ and $600 \mathrm{rpm}$, and its pole number, $n_{p}=12$, the electrical frequency of the PMSG, $f_{e}=n_{m} \cdot n_{p} / 12$, ranges between: $f_{e}=15$ and $60 \mathrm{~Hz}$. The $P I$ compensator is designed to obtain a crossover frequency of the loop gain less than $f_{e} / 2$, with a phase margin higher than $50^{\circ}$. 
It is worth to point out that as $V_{d}$ is proportional to $f_{e}$, the crossover frequency also varies with $f_{e}$, so that the design of the PLL PI compensator is not straightforward.

Fig. 4 shows the loop gain of the SRF-PLL that allows to obtain a phase margin higher than $50^{\circ}$ in the generator speed range. The gains of the PLL PI controller are: $K_{p}=0.22$ and $K_{i}=30 \mathrm{rad} / \mathrm{s}$.

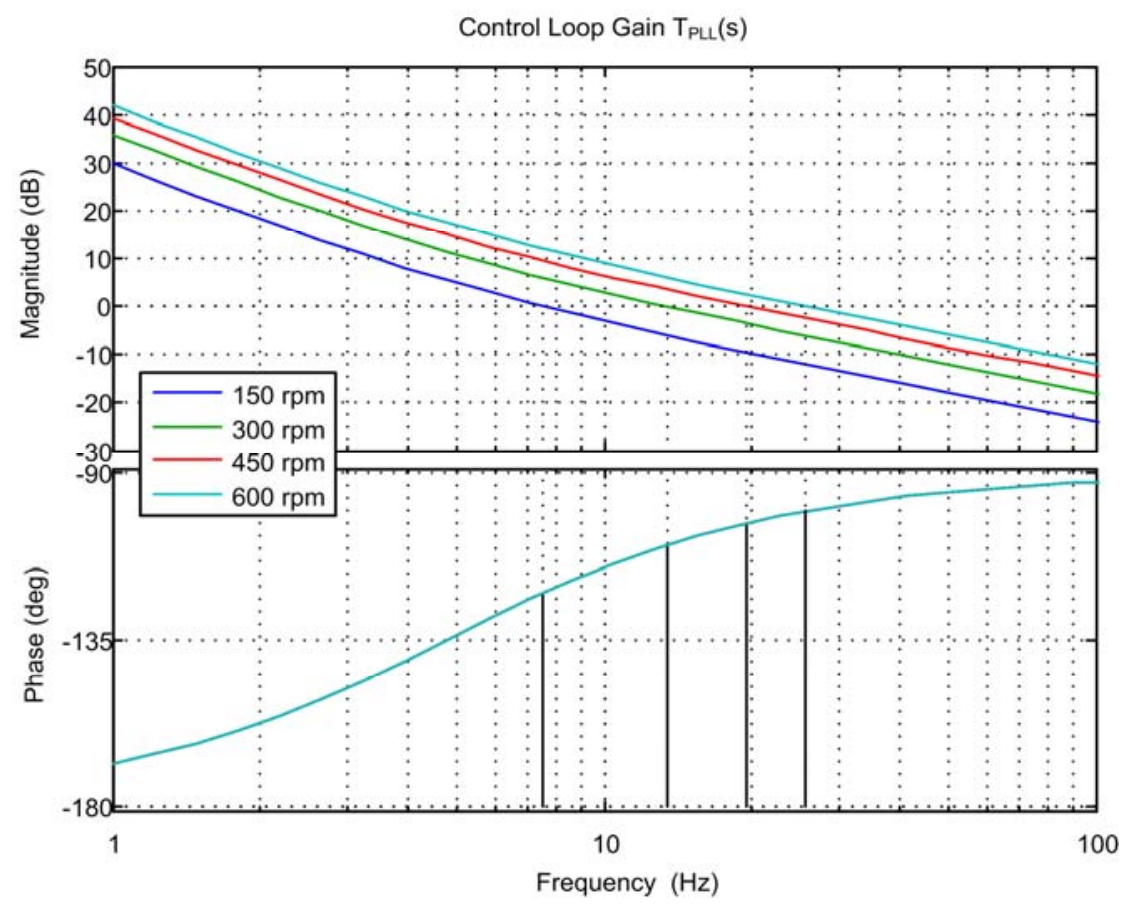

Fig. 4. Bode diagram (module and phase) of the SRF-PLL Loop Gain Transfer Function

Note that the Bode diagrams of the SRF-PLL loop gain strongly depend on the PMSG output voltage and therefore on the turbine speed. To avoid that, the SRF-PLL may be implemented by previously normalizing the PMSG voltages. This allows that the value of $V_{d}$ doesn't depend on the speed of the generator, so that it is possible to easily fulfill the required phase margin. The normalization of the PMSG voltages is defined following (2)

$$
V_{a n}=\frac{V_{a}}{\sqrt{V_{a}^{2}+V_{b}^{2}}} \quad ; \quad V_{b n}=\frac{V_{b}}{\sqrt{V_{a}^{2}+V_{b}^{2}}}
$$

In this case, the gains of the PLL PI controller are: $K_{p}=70$ and $K_{i}=4200 \mathrm{rad} / \mathrm{s}$. With the proposed approach, both the crossover frequency of the SRF-PLL loop and the phase margin is fixed in the whole range of the turbine speeds.

\section{Speed estimation starting from the dc current and voltage of the Boost Rectifier}

The speed estimation starting from the measurement of both the current and the voltage at the Boost Rectifier dc output follows the scheme shown by Fig. 5. 


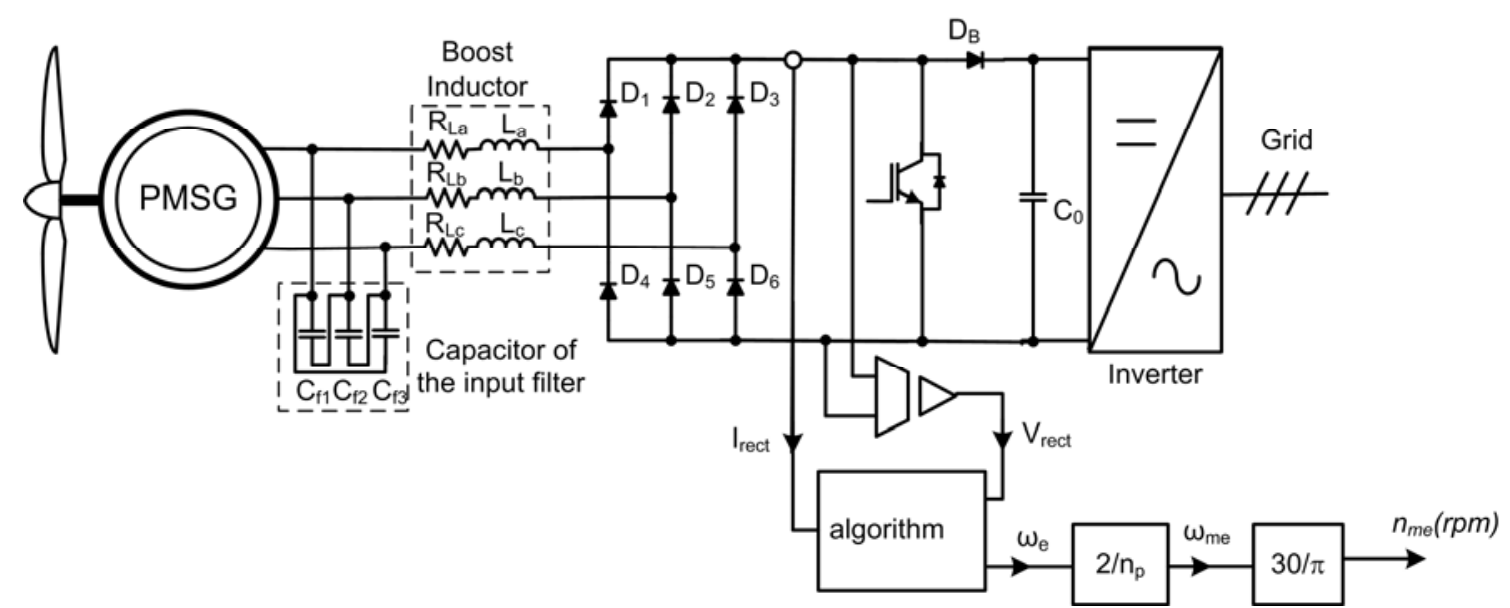

Fig. 5. Scheme of the Speed Estimation starting from the measurement of the currents and voltages at the Boost Rectifier.

By analyzing the behavior of the rectifier [10], it is derived that the rotational frequency of the PMSG can be expressed by (3)

$$
\omega_{e}=\frac{\frac{\pi V_{\text {rect }}}{3}+\left(R_{g}+R_{L}\right) I_{\text {rect }}}{\left(\frac{2 \sqrt{2} K_{\text {fem }}}{n_{p}}-\left(L_{g}+L\right) I_{\text {rect }}\right)}
$$

where $I_{\text {rect }}$ and $V_{\text {rect }}$ are the current and voltage at the output of the diode bridge.

The drawback of this technique is that it depends on the characteristics of the boost rectifier and of the generator, which can change due to components aging, temperature, etc., producing estimation errors. Besides, the main advantage compared to other techniques is that additional sensors are not required, because the measured signals are also needed for other functions of the control structure.

\section{Speed Estimation using Extended Kalman Filter.}

The Extended Kalman Filter (EKF) has been extensively used in speed and position estimation of permanent magnet synchronous motors [11]-[12] and wind generators [13]. In the following, the fundamentals of the Kalman Filter concept are summarized and applied to the WEGS under study.

\subsection{Extended Kalman Filter Concept.}

The discrete state formulation of the EKF [11]-[12] is defined by (4)

$$
\begin{gathered}
x(k+1)=A_{k} x(k)+B_{k} u(k)+\sigma(k) \\
y(k)=C_{k} x(k)+\mu(k)
\end{gathered}
$$

where $x$ is the estimated signal vector, $u$ is the deterministic input vector and $y$ is the output vector. $\sigma(\mathrm{t})$ and $\mu(\mathrm{t})$ represent the process and measurement zero-mean Gaussian noises, respectively. The noise measurement are independent of the system state $x . \mathrm{Q}$ and $\mathrm{R}$ will denote the covariance matrixes of $\sigma(\mathrm{t})$ and $\mu(\mathrm{t})$, respectively. $\mathrm{P}$ is defined as the covariance matrix of the estimation error.

The EKF algorithm has two steps: estimation and correction. The first step sets a prediction of the state estimation $(x 1)$ and of its covariance matrix $(P 1)$. This is implemented by means of the following recursive relationships: 


$$
\begin{gathered}
x 1(k+1)=A_{k} \hat{x}(k)+B_{k} u(k) \\
P 1(k+1)=F(k+1) \hat{P}(k) F^{T}(k+1)+Q
\end{gathered}
$$

where $F(k+1)$ is expressed by $(7)$.

$$
F(k+1)=\frac{\partial\left[A_{k} \hat{x}(k)+B_{k} u(k)\right]}{\partial x}
$$

The second step corrects the prediction of the state estimation and that of its covariance matrix by feedback of the measured quantities. This is performed by means of the following recursive relationships:

$$
\begin{gathered}
\hat{x}(k+1)=x 1(k+1)+K(k+1)\left[y(k+1)-C_{k}(k+1) x 1(k+1)\right] \\
\hat{P}(k+1)=P 1(k+1)-K(k+1) H(k+1) P(k+1)
\end{gathered}
$$

where $H(k+1)$ is the gain matrix of the filter, being defined by $(10)$.

$$
K(k+1)=P(k+1) H^{T}(k+1)\left[H(k+1) P(k+1) H^{T}(k+1)+R\right]^{-1}
$$

In (10), matrix $H(k+1)$ is determined by (11).

$$
H(k+1)=\frac{\partial\left[C_{k} x(k)\right]}{\partial x}
$$

In this application, the EKF can be implemented following two different approaches. The first one starts from the electromotive force of the PMSG, being the knowledge of the PMSG parameters necessary. The second approach directly uses the PMSG output voltage; the advantage is that it isn't necessary to know the PSMG model or the PSMG characteristics.

\subsection{Permanent Magnet Synchronous Generator Model.}

The equivalent electrical circuit of the PMSG is shown in Fig. 6.

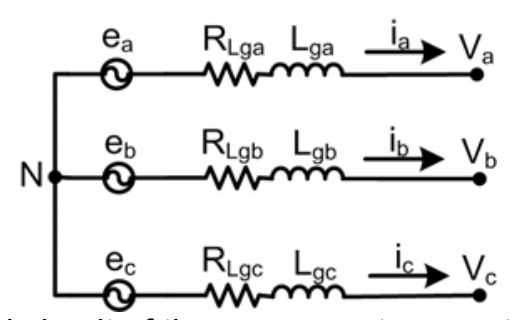

Fig. 6. Equivalent electrical circuit of the permanent magnet synchronous generator.

$V_{a}, V_{b}$ and $V_{c}$ are the voltages at the generator output, and $e_{a}, e_{b}$ and $e_{c}$ are the electromotive forces produced by the generator, being expressed by (12)

$$
\begin{gathered}
e_{a}=K_{f e m} \omega_{e} \sin \left(\omega_{e} t\right) \\
e_{b}=K_{f e m} \omega_{e} \sin \left(\omega_{e} t-\frac{2 \pi}{3}\right) \\
e_{c}=K_{f e m} \omega_{e} \sin \left(\omega_{e} t+\frac{2 \pi}{3}\right)
\end{gathered}
$$

The PMSG electrical model can be expressed in the state space, as it is shown by (13). 


$$
\left[\begin{array}{l}
V_{a} \\
V_{b} \\
V_{c}
\end{array}\right]=-R_{g}\left[\begin{array}{l}
i_{a} \\
i_{b} \\
i_{c}
\end{array}\right]-L_{g} \frac{d}{d t}\left[\begin{array}{l}
i_{a} \\
i_{b} \\
i_{c}
\end{array}\right]+\left[\begin{array}{l}
e_{a} \\
e_{b} \\
e_{c}
\end{array}\right]
$$

The EKF estimator works with the PMSG modeled in a synchronous reference frame [14], which is depicted in Fig. 7, where $V_{d}$ and $V_{q}$ are the output voltages in the synchronous $d q$ reference frame, and $i_{d}$ and $i_{q}$ are the generator output currents in the $d q$ frame.

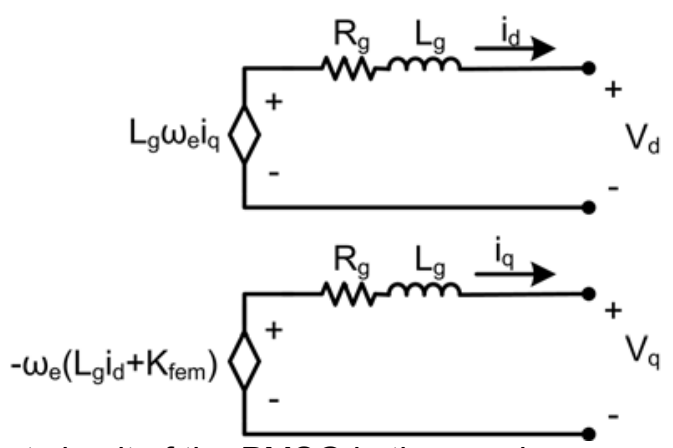

Fig. 7. Equivalent circuit of the PMSG in the synchronous reference frame.

The state space equations of the PMSG in the $d q$ synchronous reference frame are expressed by (14).

$$
\begin{gathered}
V_{d}=-R_{g} i_{d}+L_{g} \omega_{e} i_{q}-L_{g} \omega_{e} i_{d} \\
V_{q}=-R_{g} i_{q}-L_{g} \omega_{e} i_{d}-L_{g} \omega_{e} i_{q}-\sqrt{\frac{3}{2}} K_{f e m} \omega_{e}
\end{gathered}
$$

\subsection{Estimation using the Electromotive Force.}

The scheme of the speed estimator using the electromotive force is shown by Fig. 8 .

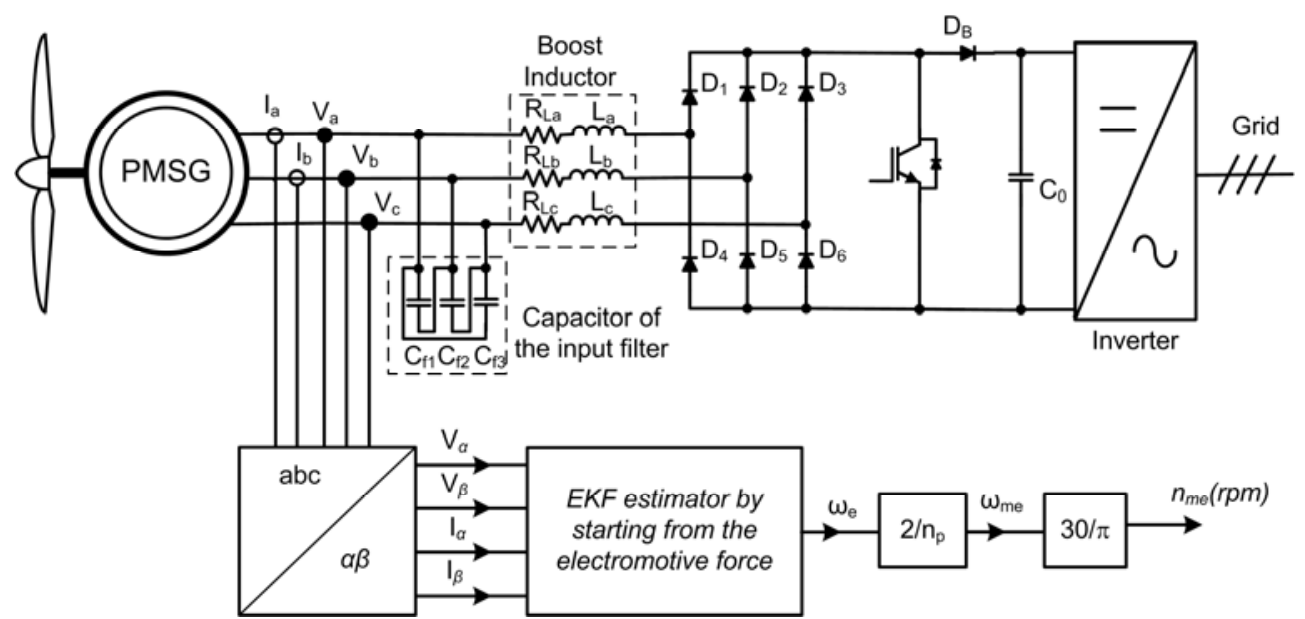

Fig. 8. Scheme of the speed estimator EKF using the electromotive force.

The values of $x, u$ and $y$ that are applied to (4) are defined by (15). Note that $y$ and $u$ are the measurement signals, represented in the stationary reference frame $(\alpha \beta)$, as shown by (16). Equation (17) shows the relationship between the currents expressed in the synchronous reference frame $(d q)$ and the stationary frame $(\alpha \beta)$. 


$$
\begin{gathered}
x=\left[\begin{array}{c}
i_{d} \\
i_{q} \\
\omega_{e} \\
\theta
\end{array}\right], \quad y=\left[\begin{array}{l}
i_{\alpha} \\
i_{\beta}
\end{array}\right], \quad u=\left[\begin{array}{l}
v_{\alpha} \\
v_{\beta}
\end{array}\right] \\
{\left[\begin{array}{l}
i_{\alpha} \\
i_{\beta}
\end{array}\right]=\sqrt{\frac{2}{3}}\left[\begin{array}{ccc}
1 & -\frac{1}{2} & -\frac{1}{2} \\
0 & \frac{\sqrt{3}}{2} & -\frac{\sqrt{3}}{2}
\end{array}\right]\left[\begin{array}{c}
i_{a} \\
i_{b} \\
i_{c}
\end{array}\right] ;\left[\begin{array}{l}
v_{\alpha} \\
v_{\beta}
\end{array}\right]=\sqrt{\frac{2}{3}}\left[\begin{array}{ccc}
1 & -\frac{1}{2} & -\frac{1}{2} \\
0 & \frac{\sqrt{3}}{2} & -\frac{\sqrt{3}}{2}
\end{array}\right]\left[\begin{array}{l}
v_{a} \\
v_{b} \\
v_{c}
\end{array}\right]} \\
{\left[\begin{array}{l}
i_{d} \\
i_{q}
\end{array}\right]=\left[\begin{array}{cc}
\cos \theta & \sin \theta \\
-\sin \theta & \cos \theta
\end{array}\right]\left[\begin{array}{l}
i_{\alpha} \\
i_{\beta}
\end{array}\right]}
\end{gathered}
$$

$\theta$ is the estimated rotor position and $\omega_{e}$ is the estimated angular frequency. The discrete matrixes of the system are expressed by (18). They depend on the sampling time of the digital control system $\left(T_{S}\right)$.

$$
\begin{gathered}
A_{k}=\left[\begin{array}{cccc}
1-\frac{T_{s} R_{g}}{L_{g}} & \omega_{e} T_{s} & 0 & 0 \\
-\omega_{e} T_{s} & 1-\frac{R_{g} T_{s}}{L_{g}} & -\sqrt{\frac{3}{2}} K_{f e m} \omega_{e} & 0 \\
0 & 0 & 1 & 0 \\
0 & 0 & T_{s} & 1
\end{array}\right] \\
B_{k}=\left[\begin{array}{cc}
-\frac{T_{s} \cos \theta}{L_{g}} & -\frac{T_{s} \sin \theta}{L_{g}} \\
\frac{T_{S} \sin \theta}{L_{g}} & -\frac{T_{s} \cos \theta}{L_{g}} \\
0 & 0 \\
0 & 0
\end{array}\right] \\
C_{k}=\left[\begin{array}{cccc}
\cos \theta & -\sin \theta & 0 & 0 \\
\sin \theta & \cos \theta & 0 & 0
\end{array}\right]
\end{gathered}
$$

In this work the covariance matrixes $Q$ and $R$ are chosen by a trial and error procedure to find the best tradeoff between filter stability and convergence time. The chosen values of $Q$ and $R$ are shown by (19).

$$
Q=\left[\begin{array}{cccc}
0.5 & 0 & 0 & 0 \\
0 & 0.5 & 0 & 0 \\
0 & 0 & 2 & 0 \\
0 & 0 & 0 & 0.01
\end{array}\right] ; R=\left[\begin{array}{ll}
1 & 0 \\
0 & 1
\end{array}\right]
$$

\subsection{Estimation starting from the generator output voltages.}

In the speed estimator starting from the measurement of the PMSG output voltages the values of $x, u$ and $y$ are expressed by (20). In this case, the measurement $(y)$ and the output $(u)$ signals agree. Both signals are represented in the stationary reference frame, following (21).

$$
x=\left[\begin{array}{c}
v_{d} \\
v_{q} \\
\omega_{e} \\
\theta
\end{array}\right], \quad y=u=\left[\begin{array}{c}
v_{\alpha} \\
v_{\beta}
\end{array}\right]
$$




$$
\left[\begin{array}{l}
v_{d} \\
v_{q}
\end{array}\right]=\left[\begin{array}{ll}
\cos \theta & \sin \theta \\
\sin \theta & \cos \theta
\end{array}\right]\left[\begin{array}{l}
v_{\alpha} \\
v_{\beta}
\end{array}\right]
$$

The discrete matrixes of the system are expressed by (22). Note that they depend on the sampling time $\left(T_{s}\right)$.

$$
\begin{gathered}
A_{k}=\left[\begin{array}{llll}
1 & 0 & 0 & 0 \\
0 & 1 & 0 & 0 \\
0 & 0 & 1 & 0 \\
0 & 0 & T_{s} & 1
\end{array}\right] \\
B_{k}=\left[\begin{array}{cc}
T_{S} \cos \theta & T_{s} \sin \theta \\
-T_{S} \sin \theta & T_{S} \cos \theta \\
0 & 0 \\
0 & 0
\end{array}\right] \\
C_{k}=\left[\begin{array}{cccc}
\cos \theta & -\sin \theta & 0 & 0 \\
\sin \theta & \cos \theta & 0 & 0
\end{array}\right]
\end{gathered}
$$

In this case, the chosen values for $Q$ and $R$ are shown by (23).

$$
Q=\left[\begin{array}{cccc}
0.5 & 0 & 0 & 0 \\
0 & 0.5 & 0 & 0 \\
0 & 0 & 2 & 0 \\
0 & 0 & 0 & 0.01
\end{array}\right] ; R=\left[\begin{array}{ll}
1 & 0 \\
0 & 1
\end{array}\right]
$$

The scheme of the speed estimator starting from the generator output voltages is shown by Fig. 9 .

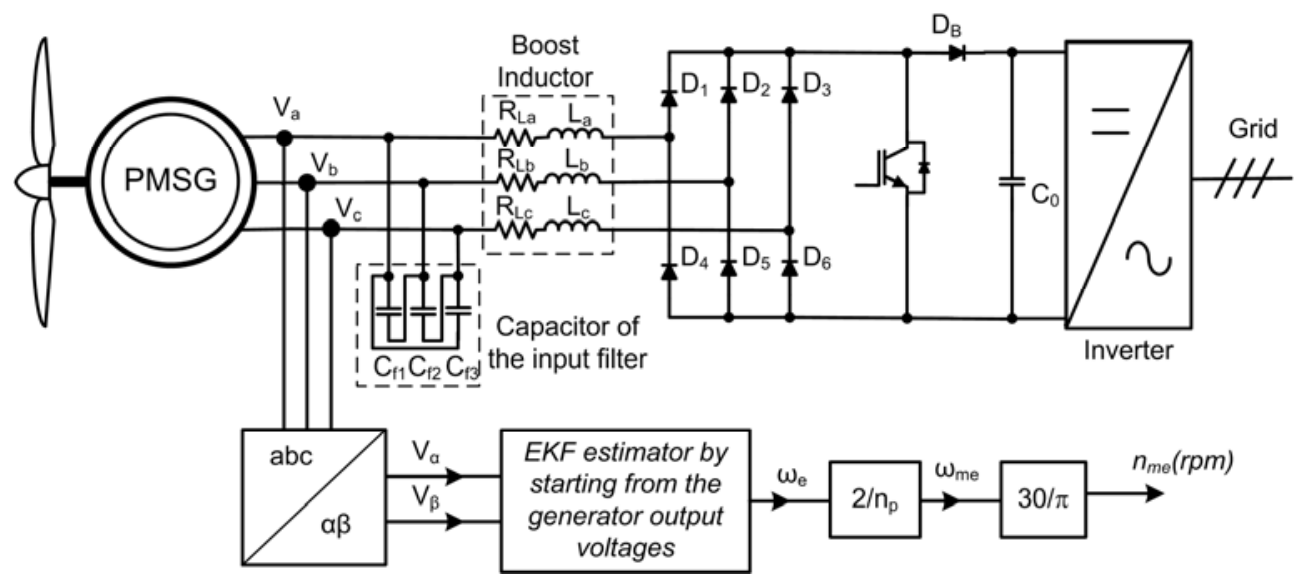

Fig. 9. Scheme of the speed estimator EKF starting from the generator output voltages.

\section{Speed Estimation by means of the Linear Kalman Filter}

In the EKF it is calculated a variable gain matrix $K(10)$ which requires a high computational cost. That may compromise its practical implementation. To reduce the computations, EKF can be simplified if the filter structure is known [15]-[17], leading to the so-called Linear Kalman Filter (LKF).

In the LKF, the state vector is defined by (24).

$$
x=\left[\begin{array}{c}
\theta \\
\omega_{e} \\
\rho
\end{array}\right]
$$


The expressions for the rotor position, $\theta(k)$, and for the angular frequency, $\omega_{e}(k)$, are given by (25)

$$
\begin{gathered}
\theta(k+1)=\theta(k)+T_{s} \omega_{e}(k) \\
\omega_{e}(k+1)=\omega_{e}(k)+\dot{\rho}(k) \\
\dot{\rho}(k+1)=\dot{\rho}(k)+\rho(k)
\end{gathered}
$$

where $\rho(k)$ is the zero-mean process Gaussian noise [15].

For the analysis of the simplified estimator the output variables are the output voltages of the PMSG in the stationary reference frame, as shown by (26).

$$
\left[\begin{array}{l}
y_{1}(k) \\
y_{2}(k)
\end{array}\right]=\left[\begin{array}{l}
V_{\alpha} \\
V_{\beta}
\end{array}\right]
$$

The LKF estimator extracts the speed information from the fundamental component of the PMSG output voltages, whereas other harmonic components are considered as measurement noise. Note that this approach is suitable for this application, because the output voltages contain both low frequency harmonics and switching frequency noise. The output variables are expressed in normalized form as shown by (27)

$$
\left[\begin{array}{l}
y_{1}(k) \\
y_{2}(k)
\end{array}\right]=\left[\begin{array}{l}
\cos \theta(k) \\
\sin \theta(k)
\end{array}\right]+\left[\begin{array}{l}
\mu_{1}(k) \\
\mu_{2}(k)
\end{array}\right]
$$

where both $\mu_{1}$ and $\mu_{2}$ represent switching noise and high-order low frequency harmonics. Considering the state space representation (25), the state vector (24), and the input $u(k)=0$ in (4), it is obtained (28):

$$
\begin{gathered}
x(k+1)=A_{s} x(k)+G_{s} \rho(k) \\
y(k)=C_{s} x(k)+\mu(k)
\end{gathered}
$$

The expression of $A_{s}, G_{s}$ and $C_{s} x(k)$ matrixes are given by (29) and (30):

$$
\begin{gathered}
A_{s}=\left[\begin{array}{ccc}
1 & T_{s} & 0 \\
0 & 1 & 1 \\
0 & 0 & 1
\end{array}\right] ; G_{s}=\left[\begin{array}{lll}
0 & 0 & 0 \\
0 & 0 & 0 \\
0 & 0 & 1
\end{array}\right] \\
C_{S} x(k)=\left[\begin{array}{c}
\cos \theta(k) \\
\sin \theta(k)
\end{array}\right]
\end{gathered}
$$

Applying (11) to (30) it results (31).

$$
H(k+1)=\frac{\partial C_{s} x(k)}{\partial x}=\left[\begin{array}{ccc}
-\sin \theta(k) & 0 & 0 \\
\cos \theta(k) & 0 & 0
\end{array}\right]
$$

However, to simplify $H(k+1)$ the $d q$ transformation matrix, $T$, can be used, following (32) and (33)

$$
\begin{gathered}
T=\left[\begin{array}{cc}
\cos \theta & -\sin \theta \\
\sin \theta & \cos \theta
\end{array}\right] \\
H(k+1)=\left[\begin{array}{cc}
\cos \theta & \sin \theta \\
-\sin \theta & \cos \theta
\end{array}\right]^{T}\left[\begin{array}{lll}
0 & 0 & 0 \\
1 & 0 & 0
\end{array}\right]
\end{gathered}
$$

where

$$
H_{s}=\left[\begin{array}{lll}
0 & 0 & 0 \\
1 & 0 & 0
\end{array}\right]
$$


Applying (32) to (10) it is obtained the simplified filter gain, $K$, following (35)

$$
K(k+1)=\left[\begin{array}{ll}
0 & K_{s 1} \\
0 & K_{s 2} \\
0 & K_{s 3}
\end{array}\right]\left[\begin{array}{cc}
\cos \theta(k) & -\sin \theta(k) \\
\sin \theta(k) & \cos \theta(k)
\end{array}\right]
$$

$K_{S 1}, K_{S 2}$ y $K_{S 3}$ are the LKF gains which are pre-calculated using the Matlab DLQE (A,G,C,Q,R) command [18], with input matrixes: $A=A s, G=R=G_{s}, C=H_{s}$ and $Q$ :

$$
Q=\lambda\left[\begin{array}{ll}
0 & 0 \\
1 & 0
\end{array}\right]
$$

In (36) the parameter $\lambda$ allows to adjust the noise rejection ability, being chosen by trial and error.

Starting from (8), it is obtained the estimation error expressed by (37)

$$
K(k+1) \cdot\left[y(k+1)-C_{s} x(k)\right]=\left[y_{2}(k) \cos \theta(k)-y_{1}(k) \sin \theta(k)\right]\left[\begin{array}{l}
K_{s 1} \\
K_{s 2} \\
K_{s 3}
\end{array}\right]
$$

Finally, the LKF equations are expressed by (38).

$$
\begin{gathered}
\varepsilon(k)=V_{\beta}(k) \cos \theta(k)-V_{\alpha}(k) \sin \theta(k) \\
\theta(k+1)=\theta(k)+T_{s} \omega_{e}(k)+K_{s 1} \varepsilon(k) \\
\omega_{e}(k+1)=\omega_{e}(k)+\rho(k)+K_{s 2} \varepsilon(k) \\
\dot{\rho}(k+1)=\dot{\rho}(k)+K_{s 3} \varepsilon(k)
\end{gathered}
$$

For this application the following values of the LKF algorithm result: $\lambda=5 \times 10^{6}, K_{s 1}=0.0032896$, $K_{s 2}=0.54221$ and $K_{s 3}=0.00044647$. It is worth pointing out that the LKF gains are independent of the PMSG parameters and could be used with a different PMSG. Another advantage of the LKF speed estimator is that the only measurement needed is that of the output voltages of the PMSG, reducing the cost of the sensors in the system. Fig. 10 shows the scheme of the LKF speed estimator.

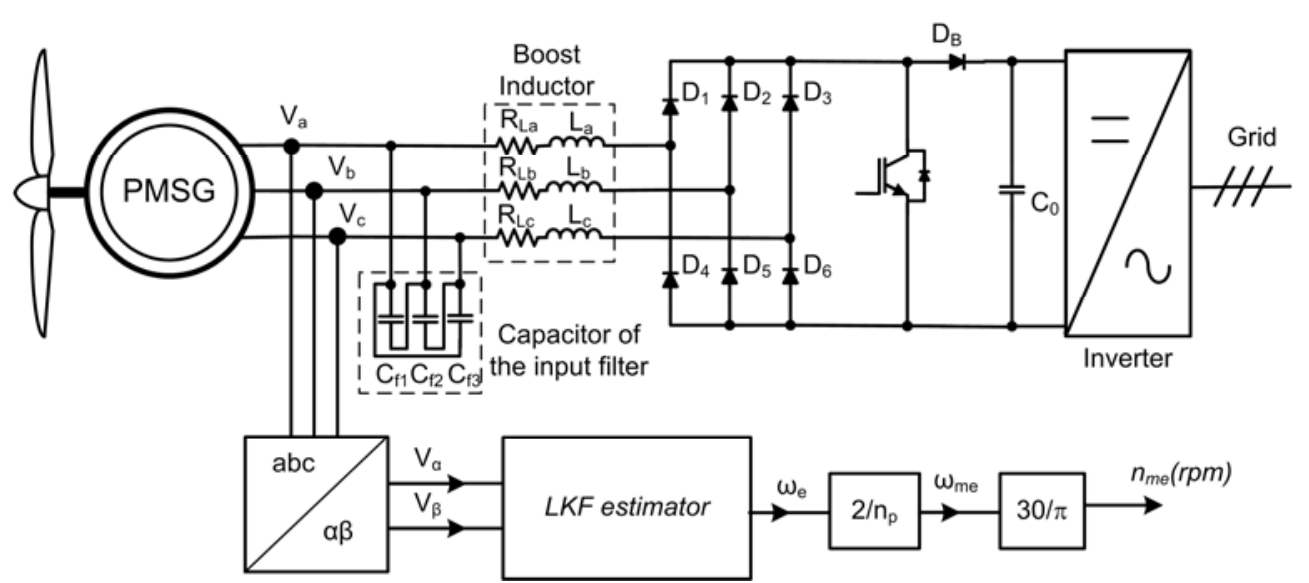

Fig. 10. Scheme of the speed estimator using a Linear Kalman Filter.

\section{RESULtS}

After the design and analysis of the speed estimators under study, their performance has been evaluated by means of accurate PSIM ${ }^{\mathrm{TM}} 7.0 .5$ simulations [19].

Fig. 11 depicts the response of the studied speed estimators to steps of the actual speed in the whole operation range. As shown by Fig. 11, not all of them provide an accurate steady-state speed estimation. For instance, note that both the estimator starting from the dc current and 
voltage of the rectifier and the EKF starting from the electromotive force failed in estimation the actual speed for some operation points. The bad performance of such estimators is mainly attributed to both the switching noise and the low frequency harmonics present in the currents and voltages of the generator (see Fig. 2). The other estimators work reasonably well in steady state.

It is worth to point out the ripple that appears in the estimation signal of some estimators. The peak to peak ripple produced by each one of the estimators is a factor that has been measured and taken into account to perform the comparative study. In the case of the PLL estimator, the ripple of the speed estimation signal increases as the generator speed does. However, when the voltages at the PLL input are normalized, it stays the same ripple value in the whole generator speed range. For that reason, this solution is preferred over the PLL without normalized input for further analysis.

In all cases, the ripple should be removed without significantly delaying the estimation, which is used to close the speed control loop. To achieve that, a second order low pass filter with a cut-off frequency $f_{c}=20 \mathrm{~Hz}$ has been chosen in this work.
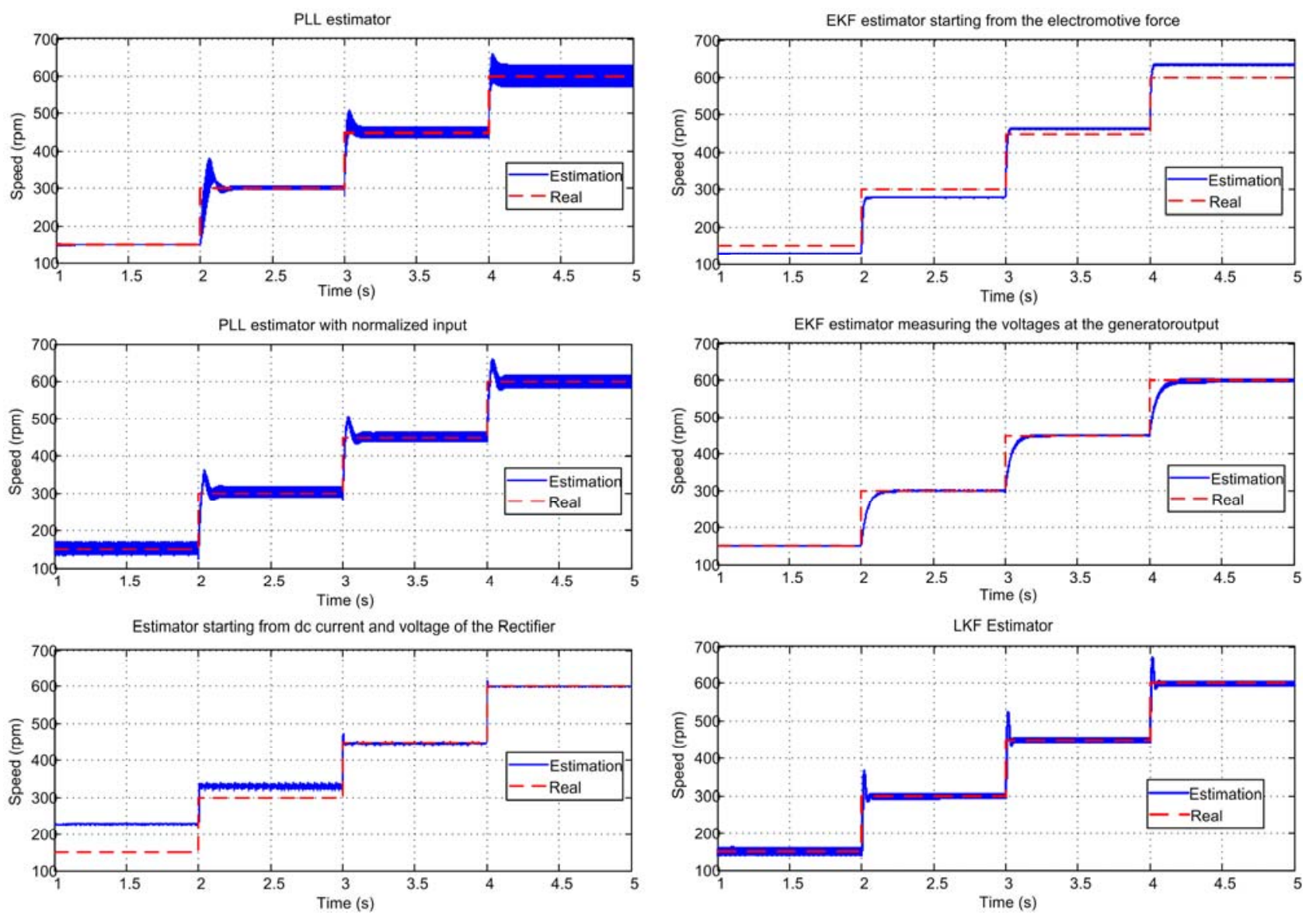

Fig. 11. The response of each of the speed estimators to speed steps.

Fig. 12 shows a detail of the speed estimation error for the three algorithms that have presented the best performance in steady state. The PLL estimator with normalized input has a maximum ripple of $\pm 15 \mathrm{rpm}$. The estimator using an EKF measuring the voltages at the generator output has a maximum ripple of $\pm 4 \mathrm{rpm}$, whereas the LKF estimator has a maximum ripple of $\pm 10 \mathrm{rpm}$. Fig. 12 also shows the error of the estimators after filtering, so that the response time of each estimator can be easily measured. The PLL estimator with normalized input has a response time of $200 \mathrm{~ms}$, the EKF estimator measuring the voltages at the generator output has a response time of $300 \mathrm{~ms}$, and the LKF estimator has a response time of $80 \mathrm{~ms}$. 

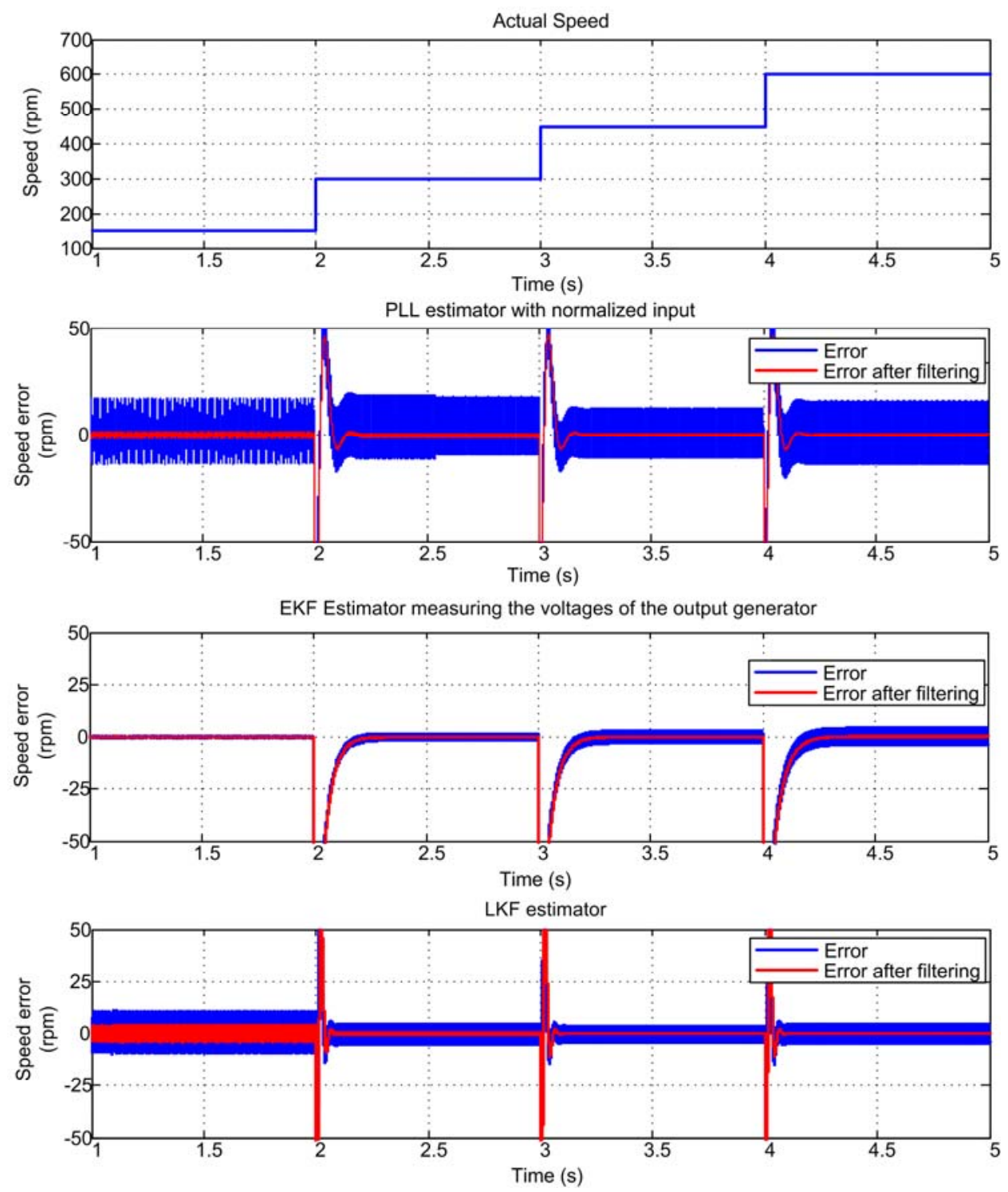

Fig. 12. Transient speed estimation error of the best steady-state estimators.

TABLE II summarizes the results of the comparative study of the different speed estimators under consideration. The best overall performance is achieved by the LKF estimator, so that it can be concluded that this technique is the most interesting one to estimate the turbine speed in small WEGS driven by means of three-phase power factor correction rectifiers in DCM.

TABLE II. Comparative of the different speed estimators studied.

\begin{tabular}{lccc}
\hline \multicolumn{1}{c}{ Estimator } & $\begin{array}{c}\text { Maximum error } \\
\text { in steady state } \\
\left(\varepsilon_{\mathrm{ss}}\right)[\mathrm{rpm}]\end{array}$ & $\begin{array}{c}\text { Maximum } \\
\text { response time } \\
\left(\mathrm{t}_{\mathrm{r}}\right)[\mathrm{ms}]\end{array}$ & $\begin{array}{c}\text { Maximum } \\
\text { Ripple [rpm] }\end{array}$ \\
\hline PLL estimator & $0 \mathrm{rpm}$ & $300 \mathrm{~ms}$ & $\pm 30 \mathrm{rpm}$ \\
$\begin{array}{l}\text { PLL estimator with normalized input } \\
\begin{array}{l}\text { Estimator starting from DC current and } \\
\text { voltage of rectifier }\end{array}\end{array}$ & $0 \mathrm{rpm}$ & $200 \mathrm{~ms}$ & $\pm 15 \mathrm{rpm}$ \\
$\begin{array}{l}\text { EKF estimator starting from the } \\
\text { electromotive force }\end{array}$ & $36 \mathrm{rpm}$ & $40 \mathrm{~ms}$ & $\pm 10 \mathrm{rpm}$ \\
$\begin{array}{l}\text { EKF estimator measuring the voltage } \\
\text { of the output generator }\end{array}$ & $0 \mathrm{rpm}$ & $100 \mathrm{~ms}$ & $\pm 4 \mathrm{rpm}$ \\
\begin{tabular}{l} 
LKF estimator \\
\hline
\end{tabular} & $0 \mathrm{rpm}$ & $300 \mathrm{~ms}$ & $\pm 4 \mathrm{rpm}$ \\
\hline
\end{tabular}




\section{CONCLUSION}

Five different speed estimators for variable speed PMSGs driven by a three-phase boost rectifier operating in discontinuous conduction mode have been designed and evaluated in this paper. The main difficulty for the design of all the estimators under study is both the switching noise and the low frequency harmonics that are present in the measurement signals. It may be concluded that the most appropriate estimator for this application is the LKF one, because it exhibits a good compromise among steady state performance, transient response time and peak to peak ripple in the estimation signal. Besides, the LKF estimator needs a lower computational effort in comparison to the great quantity of matrix operations needed by the EKF estimators.

\section{Acknowledgements}

The first author thanks the support of the Instituto Politécnico Nacional (IPN) and of the Comisión de Operación y Fomento de Actividades Académicas (COFAA) to finance his stay at the Universidad Politécnica de Valencia (UPV). This work was supported by the Spanish Ministry of Science and Innovation under Grant ENE2009-13998-C02-02.

\section{References}

[1] Jamal A. Baroudi, Venkata Dinavahi, Andrew M. Knight, "A review of power converter topologies for wind generators", Renewable Energy 32, pp. 2369-2385, 2007.

[2] Md. Arifujjaman, M.T. Iqbal, J.E. Quaicoe, "Reliability analysis of grid connected small wind turbine power electronics", Applied Energy, Volume 86, Issue 9, September 2009, Pages 1617-162.

[3] Iulian Munteanu, Antoneta Iuliana Bratcu, Emil Ceangǎ, "Wind turbulence used as searching signal for MPPT in variable-speed wind energy conversion systems", Renewable Energy, Volume 34, Issue 1, January 2009, Pages 322-32.

[4] O. Carranza, G. Garcerá, E. Figueres, L.G. González, "Peak current mode control of three-phase boost rectifiers in discontinuous conduction mode for small wind power generators", Applied Energy, Available online 7 March 2010.

[5] E. Figueres, G. Garcera, J. Sandia, F. Gonzalez-Espín, J. Calvo, "Sensitivity Study of the Dynamics of Three-Phase Photovoltaic Inverters with an LCL Grid Filter", IEEE Transactions on Industrial Electronics, Vol. 56, No. 3, March 2009, pp. 706-717.

[6] M. Ciobotaru, R. Teodorescu, F. Blaabjerg, "Control of single-stage single-Phase PV inverter", 11th European Conference on Power Electronics and Applications, EPE '05, pp. 1-10.

[7] Jemaa Brahmi, Lotfi Krichen, Abderrazak Ouali, "A comparative study between three sensorless control strategies for PMSG in wind energy conversion system", Applied Energy, Volume 86, Issue 9, September 2009, Pages 1565-157.

[8] A.M. Salamah, S.J. Finney, B.W. Williams, "Three-phase phase-lock loop for distorted utilities", IET Electric Power Applications, Vol. 1, Issue 6, Nov. 2007 pp. 937 - 945

[9] P. Rodriguez, J. Pou, J. Bergas, J.I. Candela, R.P. Burgos, D. Boroyevich, Decoupled Double Synchronous Reference Frame PLL for Power Converters Control, IEEE Transactions on Power Electronics, Vol. 22, Issue 2, March 2007, pp. $584-592$.

[10] Mohan, Undeland, Robbins, Power Electronics, Converters, Applications and Design, USA, John Wiley \& Sons, INC, 2003

[11] S. Bolognani, R. Oboe, and M. Zigliotto, "Sensorless Full-Digital PMSM Drive With EKF Estimation of Speed and Rotor Position, " IEEE trans. on Industrial Electronics, February 1999, vol. 46, no.1, pp. 184-191.

[12] Chan Tze-Fun, P. Borsje, Wang Weimin, "Application of Unscented Kalman filter to sensorless permanent-magnet synchronous motor drive", IEEE International Electric Machines and Drives Conference, IEMDC '09, 3-6 May 2009, pp. 631-638.

[13] L.G. González, E. Figueres, G. Garcerá, O. Carranza, "Maximum-power-point tracking with reduced mechanical stress applied to wind-energy-conversion-systems", Applied Energy, In Press, Corrected Proof, Available online 6 January 2010.

[14] Yin Ming, Li Gengyin, Zhou Ming, Zhao Chengyong, "Modeling of the Wind Turbine with a Permanent Magnet Synchronous Generator for Integration", IEEE Power Engineering Society General Meeting, 2007, 24-28 June 2007 pp. 1-6. 
[15] L.Harnefors, "Speed Estimation From Noisy Resolver Signal," Proceedings of the Sixth International Conference on Power Electronics and Variable Speed Drives, pp. 279-282, 1996;

[16] M.C. Huang, A.J. Moses, F. Anayi, X.G. Yao, "Linear Kalman filter (LKF) sensorless control for permanent magnet synchronous motor based on orthogonal output linear model", International Symposium on Power Electronics, Electrical Drives, Automation and Motion, 2006. SPEEDAM 2006. 23-26 May 2006, pp.1381 - 1386 S. Stasi, L.

[17] Liu Yong, Zhu Zi Qiang, D. Howe, "Instantaneous Torque Estimation in Sensorless Direct-TorqueControlled Brushless DC Motors", IEEE Transactions on Industry Applications, Vol. 42, Issue 5, Sept.Oct. 2006, pp. 1275-1283.

[18] Matlab 7.6, User's Guide (2008), MathWork Inc, February 2008.

[19] PSIM 7.0 User's Guide (2006), Powersim Inc., March 2006. 